\title{
Fortalecimiento del vínculo entre Universidades en el espacio Atlántico: Dimensión internacional en los métodos de enseñanza y aprendizaje en la educación superior
}

\author{
Strengthening the bond between Universities in Atlantic area: International \\ dimension in learning and teaching methods of Higher Education
}

\author{
Lucía Martínez-Quintana $^{1}$ \\ lucia.martinez@ulpgc.es
}

\author{
${ }^{1}$ Departamento de Arte, Ciudad y Territorio \\ Universidad de Las Palmas de Gran Canaria \\ Las Palmas de Gran Canaria, España
}

\begin{abstract}
Resumen- Conocemos los resultados positivos del uso del Aprendizaje Basado en Proyectos (ABP) y de las estrategias de evaluación formativa en la educación superior. En esta ponencia exponemos cómo el simple hecho de dar una dimensión internacional a los métodos de enseñanza y aprendizaje repercute de forma exponencial en la mejora del desarrollo de competencias profesionales. En este método didáctico ha participado Cardiff University, una de las universidades socias de la Universidad de Las Palmas de Gran Canaria que se ubican en la zona Atlántica, incorporándose en las actividades del Programa docente de la materia de "Urbanística, Ordenación de Territorio y Proyectos de Urbanismo II", que pertenece al módulo proyectual de los estudios del Grado en Arquitectura y se imparte en el $4^{\circ}$ semestre de la titulación, con una carga lectiva de 4,5 ECTS.
\end{abstract}

\section{Palabras clave: Internacional, Atlántico, Proyecto Urbano}

Abstract- It is conventional wisdom that Project-Oriented Learning (POL) and formative assessment strategies in higher education is positive. In this paper we show how the fact of giving an international dimension to this educational methodology has an exponential impact on the improvement of professional competences. Cardiff University is involved in this didactic method. This universty is one of the University of de Las Palmas de Gran Canaria's partner universities located in the Atlantic area, and it takes part in some teaching program activities applied to "Planning, Land Management and Urban Projects II". This subject is part of Undergraduate Degree in Architecture (Project module), and it is taught during the second (summer) semester to 2th year Studies (4.5 ECTS).

Keywords: International, Atlantic, Urban Project

\section{INTRODUCCIÓN}

Siendo conscientes de que cada vez estamos inmersos en un contexto más global, hacemos la apuesta por introducir en la actividad docente del Grado en Arquitectura de la Universidad de Las Palmas de Gran Canaria (ULPGC), a través de la materia que coordino: "Urbanística, Ordenación de Territorio y Proyectos de Urbanismo II", el incremento del uso de las TIC's y la implantación de actividades en su proyecto docente que aboguen por una universidad cada vez más internacionalizada.

La Universidad de Las Palmas de Gran Canaria ha conseguido situarse como una de las principales universidades del espacio Atlántico, destacando en los programas de movilidad internacional y en su estrecha implicación con las nuevas tecnologías. Por otro lado, al ocupar una posición geoestratégica en el Atlántico y promover las relaciones entre Europa, América y África Occidental, la ULPGC impulsa a que la internacionalización defina su identidad. Actualmente en nuestra universidad se imparten 42 títulos de grado presenciales y 5 on-line, 26 másteres universitarios, además de 13 programas de doctorado, 55 maestrías, expertos y formación especializada propios. Integrada por 115 grupos de $\mathrm{I}+\mathrm{D}+\mathrm{i}$ y 11 Institutos de investigación, la ULPGC ha obtenido la consideración de Campus de Excelencia Internacional en el área marino-marítima, en la convocatoria de 2010 del Ministerio de Educación.

Por ello, fortalecer lazos interuniversitarios y promover actividades docentes a través de la red global de socios en el ámbito internacional, se ha convertido en un incentivo para seguir intensificando la internacionalización de los estudios de Arquitectura en la ULPGC y lograr una formación internacional de sus egresados.

\section{CONTEXTO}

Haciendo de la internacionalización de la ULPGC una de sus fortalezas, en el año 2015 decidí establecer un convenio con Cardiff University, en Gales (Reino Unido), y desarrollar en dicha universidad una "Staff Mobility Erasmus" de más de cinco meses con fines docentes y de investigación. Al estar ubicada en la zona norte del área atlántica, vi como oportunidad el hacerla partícipe del grupo de universidades Socias de la ULPGC en el espacio Atlántico y crear vínculos.

Cardiff University es considerada una de las "top schools of architecture in the UK", y esta experiencia me permitió conocer la estructura docente del sistema educativo británico y contrastarla con el sistema español en los estudios 
universitarios de Arquitectura (Architecture) y Planeamiento (Geography and Planning). Al mismo tiempo, estreché relaciones con otros docentes de Cardiff University, pertenecientes a mi área de conocimiento.

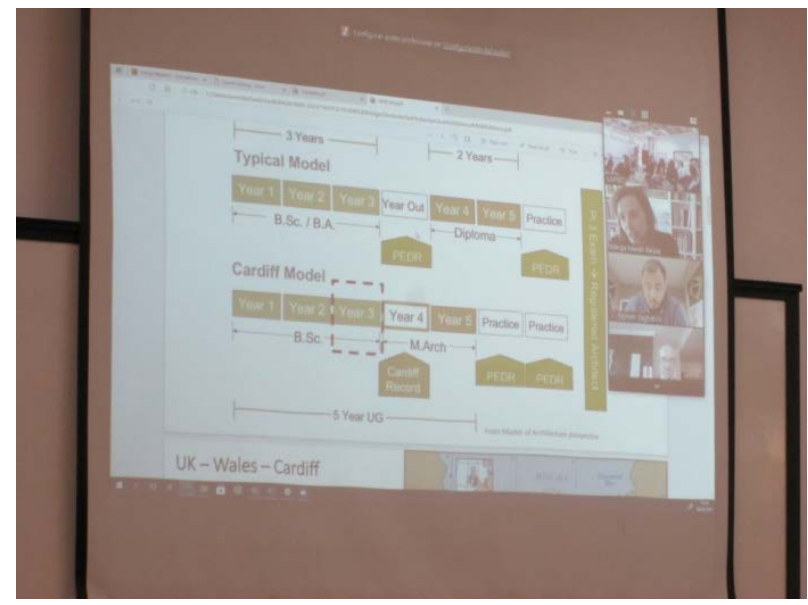

Figura 1: Una de las diapositivas del encuentro, mostrando a los participantes las diferencias entre el sistema español y el británico en los estudios universitarios de Arquitectura

Para el fortalecimiento de este vínculo entre universidades y su implantación en la titulación, también me apoyé en el hecho de que la Escuela de Arquitectura de la ULPGC desarrolla alianzas estratégicas con otras universidades, y cuenta con una Subdirección de Internacionalización y Cooperación que asesora y gestiona los diferentes programas de movilidad, tanto en el ámbito nacional (programa SICUE) como en Europa (programa Erasmus + ) o con universidades de América Latina y Estados Unidos (programa MUNDUS).

\section{A. Aplicación}

En aras de apostar por el plan de internacionalización de la ULPGC, he reforzado esta relación ULPGC-Cardiff University llevándola al espacio del aula, y aplicándola en algunas actividades docentes de la materia de "Urbanística, Ordenación de Territorio y Proyectos de Urbanismo II", la cual coordino. En su programa docente he incorporado actividades conjuntas de debate y reflexión a través de las TIC's.

El aplicar y potenciar el uso de las Tecnologías de la Información y de la Comunicación en el ámbito docente, busco la ampliación de horizontes como productor de conocimiento útil. Porque lo imprescindible en la educación superior es que el acto de la educación tienda a "enseñar a aprender", no centrándose exclusivamente en el desarrollo de la memoria, sino en la mejora de la inteligencia crítica, en el "saber hacer" (hecho que en la actualidad apuesta esencialmente a preparar para el mundo profesional), y en la apertura hacia nuevas realidades (Zabala y Arnau, 2014, p. 149)

Hemos constatado que estas nuevas realidades pueden llegar a los estudiantes a través del debate y la reflexión con otras universidades socias y partícipes de un mismo espacio común, el área Atlántica. Induciendo al estudiante a conocer otros procedimientos de análisis territorial y resolución proyectual en el ámbito urbano, haciéndolos sensibles y receptivos a otras culturas, a otros procedimientos, y haciéndolos tolerantes con las diferencias. Según Pujolas
(2008), el aprendizaje cooperativo es el marco ideal para responder a tres urgencias educativas: la educación para el diálogo, la educación para la convivencia y la educación para la solidaridad.

\section{B. Objetivos}

Con la aplicación de esta metodología educativa se busca:

Fortalecer y mejorar los procesos metodológicos del aprendizaje entre universidades y garantizar una adaptabilidad a las nuevas tecnologías educativas en la formación de nuestro alumnado.

Intensificar la internacionalización de los estudios de Grado en Arquitectura de la ULPGC, a través de la red global de socios, al incorporarlos en algunas actividades docentes, con el fin de que cobre especial importancia la formación internacional de los estudiantes.

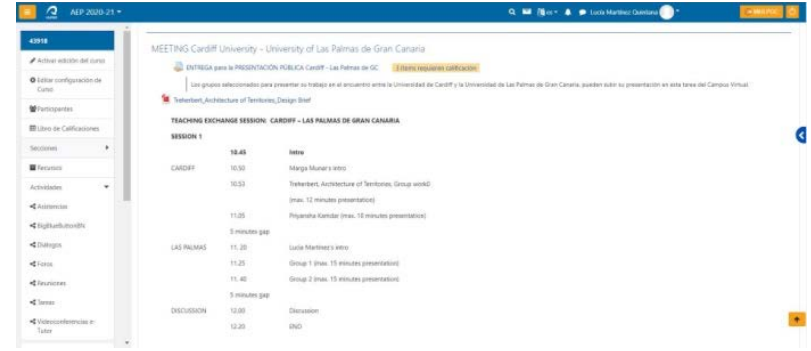

Figura 2: Publicación en el Campus Virtual de la Jornada Docente a desarrollar entre la Universidad de Cardiff y la Universidad de Las Palmas de Gran Canaria

Establecer nuevos escenarios docentes con otras universidades del espacio atlántico, que potencien la Atlanticidad y permita que la sociedad perciba a la ULPGC como una universidad verdaderamente internacionalizada.

Promover la interacción entre nuestros estudiantes y el de otras universidades, a través de actividades específicas desarrolladas en la docencia, fomentando así la participación activa y la acreditación de la competencia lingüística de todos los participantes en aras del plan de internacionalización de la ULPGC.

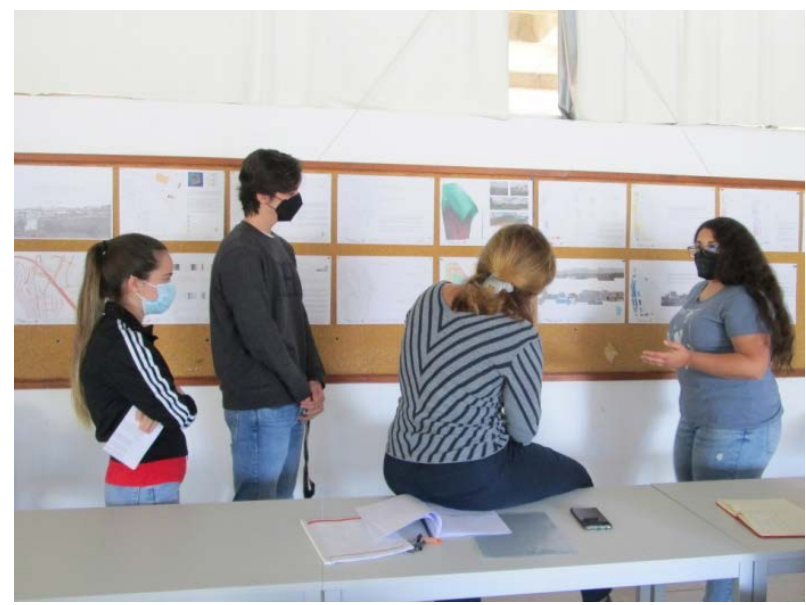

Figura 3: Defensa pública de los trabajos ante el resto de compañeros

Apostar por una transferencia de conocimientos intercultural que permita al estudiante conocer nuevas realidades, otros modos de hacer, y desarrollar en ellos la 
inteligencia crítica. El planeamiento, “aunque a menudo se forma en respuesta a problemas muy particulares en contextos específicos, siempre se ha extendido más allá de las fronteras" (Friedmann, 2010, p.313).

\section{DESCRIPCIÓN}

La materia de "Urbanística, Ordenación del Territorio y Proyectos de Urbanismo II" está adscrita al Departamento de Arte, Ciudad y Territorio, perteneciendo al módulo proyectual de los estudios del Grado en Arquitectura de la ULPGC. Se imparte en el $4^{\circ}$ semestre de la titulación, con una carga lectiva de 4,5 créditos ECTS. En ella, el estudiante afronta la concepción, la práctica y el desarrollo de proyectos urbanos de pequeña escala y adquiere la capacidad para diseñar y ejecutar trazados urbanos elementales. En su desarrollo, el estudiante aborda el medio físico, el medio social y la teoría e historia de los trazados urbanos, donde debe adquirir el conocimiento adecuado de las tradiciones arquitectónicas, urbanísticas y paisajísticas del espacio atlántico, y de la cultura canaria y occidental. En definitiva, al estudiante se le introduce en el concepto de "sitio", entendiendo "no solamente el entorno mismo donde puede ser construido un edificio, sino también el lugar geográfico donde está situado, el medio geológico que lo rodea" (Luçart, 1953, p. 495).

Las competencias asignadas a esta materia son cuatro comunes al Grado (CG1; CG6; CG7 y CG8) y dos específicas del módulo Proyectual (CY2 y CY12)

CG1 Capacidad de análisis y síntesis

\section{CG6 Resolución de problemas}

\section{CG7 Toma de decisiones}

\section{CG8 Razonamiento crítico}

CY2 Aptitud para la concepción, la práctica y desarrollo de proyectos urbanos.

CY12 Capacidad para diseñar y ejecutar trazados urbanos elementales.

Y los objetivos marcados en el aprendizaje son cuatro, todos ellos interrelacionados con las competencias mencionadas anteriormente:

OB1.- Tener capacidad para distinguir la ciudad compacta de la ciudad de planta abierta. (CG1)

OB2.- Alcanzar la capacidad de lectura de las deficiencias y distorsiones de un tejido o un fragmento de tejido urbano. (CG 6; CG8)

OB3.- Adquirir un nivel de cultura disciplinar, tanto teórica como proyectual, para ser capaz de dar respuesta a una problemática urbana. (CG 7; CY2)

OB4.- Abordar, mediante el proyecto urbano, una repuesta que reequilibre la mala conformación de un pequeño fragmento de ciudad. (CG 7; CG8; CY 12)

\section{A. Metodología}

Con el desarrollo de una metodología didáctica orientada en el Aprendizaje Basado en Proyectos, el estudiante deberá asimilar los contenidos, tanto teóricos como prácticos, de las clases impartidas. Para ello, el estudiante aborda los conceptos sobre el proyecto urbano y la ciudad con el objeto de profundizar en la reflexión y en el debate sobre los mecanismos de intervención en la ciudad a través del Proyecto Urbano.

La metodología empleada en las actividades a desarrollar afecta tanto a la enseñanza presencial como la no presencial. Con un total de 4,5 créditos ECTS que componen la asignatura, la enseñanza presencial se lleva a cabo con la intervención directa del profesor y ocupa el $50 \%$ de la carga lectiva (2,25 créditos ECTS docentes); y la enseñanza no presencial, realizada de forma autónoma por el estudiante, ocupa el otro $50 \%$ de la carga restante, la cual es bastante significativa dado que el estudiante requerirá dedicación autónoma para la asimilación de los contenidos teóricos y para el desarrollo de la parte práctica y proyectual de la asignatura.

En las ACTIVIDADES PRESENCIALES (2,25 ECTS), se desarrollan:

Clases Teóricas (0,375 ECTS). Son clases basadas en la previa lectura de diversos textos y posterior debate conjunto en clase. Deben ser entendidas como la construcción de una pequeña cultura urbana, y basan su razón en la formación teórica del estudiante, mediante el estudio y la reflexión para una comprensión básica de la ciudad, en la que cabe destacar la relación arquitectura / ciudad como una relación clave para el entendimiento del hecho urbano.

Clases Prácticas / Taller de análisis (0,675 ECTS), Consisten en la lectura del área en la que se desplegará con posterioridad la intervención proyectual. En este proceso se analiza un fragmento pequeño de ciudad con el objeto de que su estudio revele al estudiante las reglas y claves constitutivas generales del lugar. Una lectura que tenderá a desvelar las formas del espacio y de las arquitecturas, los contenidos funcionales existentes en el área de estudio y los diversos órdenes que constituyen y explican el lugar.

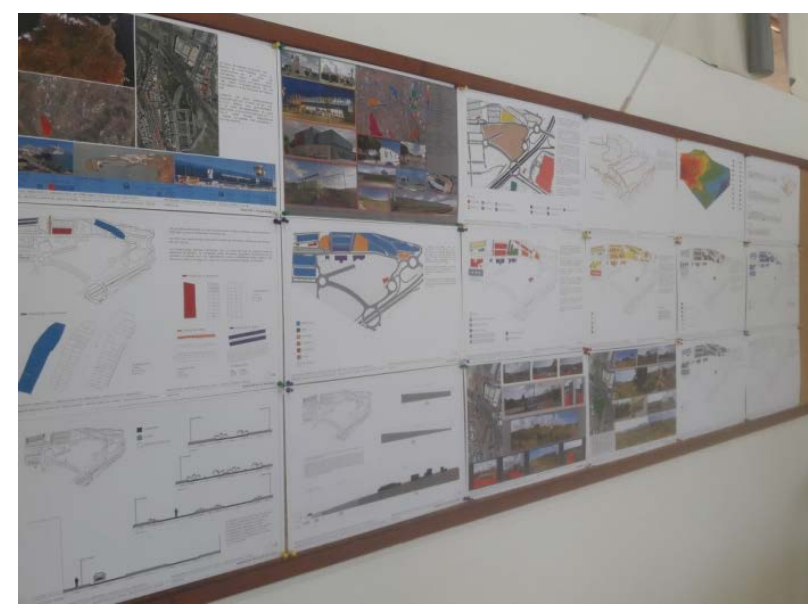

Figura 4: Exposición en el aula de los trabajos elaborados en el Taller de análisis

Clases prácticas / Taller proyectual (1,05 ECTS). Ocupa el gran peso de las actividades presenciales. En las clases proyectuales se determinará la intervención del proyecto urbano en función de las conclusiones del análisis y del programa dado. Se definirán los parámetros de forma de los diferentes espacios libres, y de las opciones tipológicas de las agrupaciones de viviendas.

Evaluación (0,15 ECTS). En la evaluación de los trabajos grupales se aplican técnicas para organizar el trabajo del 
proyecto, de manera que la participación de todos los miembros del grupo sea imprescindible para el éxito final. (Valero-García, 2007, p. 7). En esta actividad es donde entran a participar también las universidades socias, dado que es la puesta en común del hacer proyectual y donde se constatan las diversas realidades del espacio atlántico.

\section{B. Procedimiento}

Se desarrollan dos encuentros: uno al final del Taller de análisis y otro al final del Taller proyectual. El procedimiento que seguimos es el siguiente:

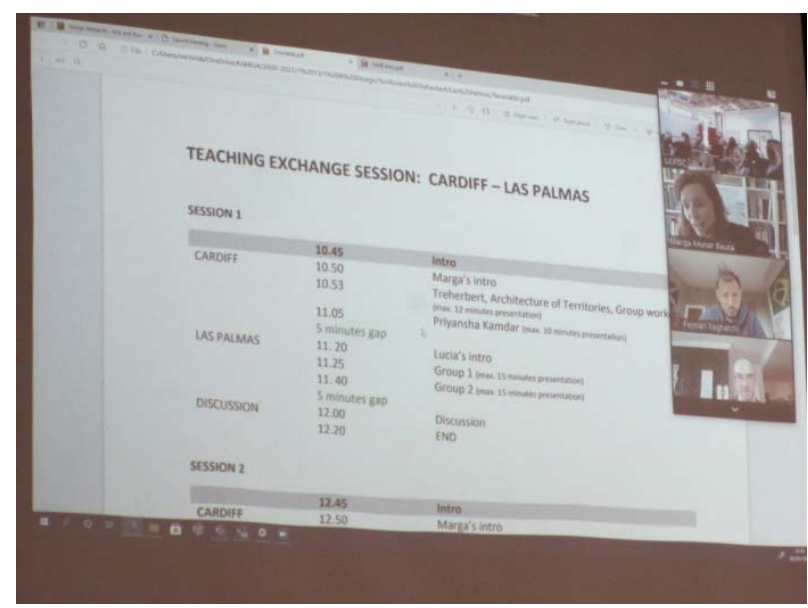

Figura 5: Estructura de una de las sesiones mantenidas en el intercambio entre la ULPGC y Cardiff University

Ambas universales pactan previamente las fechas más propias para la puesta en común, dentro de sus programas docentes.

Durante el desarrollo de las actividades docentes cada universidad socia preselecciona los proyectos más relevantes y propicios para generar discusión. El tiempo de desarrollo de esta actividad es de 3,75 horas y al disponer de un tiempo limitado, resulta imposible que sean expuestos la totalidad de los trabajos desarrollados en el curso.

Se crea el espacio virtual de trabajo colaborativo a través de las TIC's (este curso académico 2020/21 utilizamos el Zoom), estableciéndose una contraseña de acceso que se publica en el campus virtual de la asignatura, para facilitar el acceso de todos los participantes.

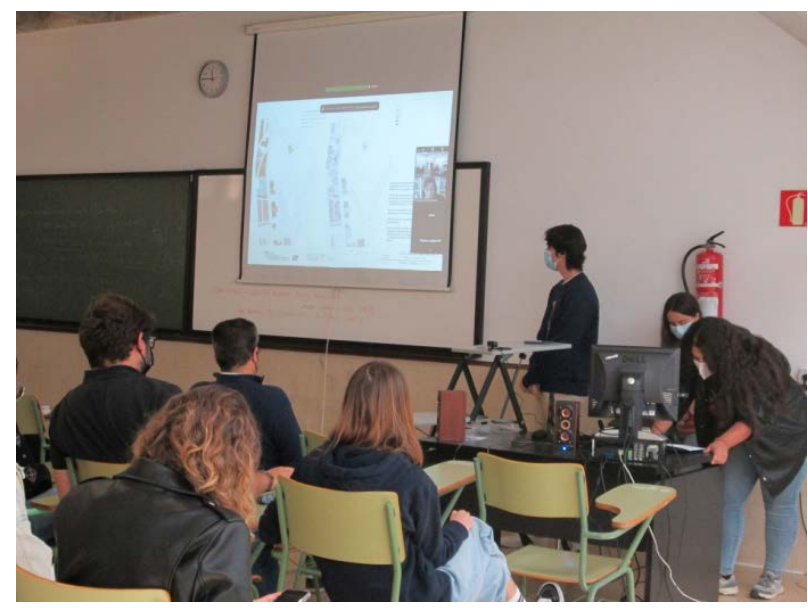

Figura 6: Presentación y defensa del trabajo de un grupo de estudiantes de la ULPGC a través de Zoom
Se desarrolla la actividad, participando tanto los estudiantes como los docentes implicados de las universidades socias. Consiste en la exposición, en lengua inglesa, del trabajo desarrollado por los alumnos preseleccionados, tanto de la fase de Análisis grupal como de la fase del Proyecto Urbano.

Esta actividad es computada en el programa docente de la asignatura como "examen y revisión", dado que el trabajo expuesto es evaluando, poniendo en énfasis sus fortalezas y debilidades.

\section{Resultados}

Por todos es sabido que la internacionalización de la Universidad exige una ampliación de horizontes y un refuerzo de alianzas entre universidades para fortalecer sus funciones esenciales como productora del conocimiento, pero por diversos motivos, no todos los estudiantes desarrollan actividades de movilidad en su etapa de formación. Por ello, provechando el gran desarrollo emergente de la TIC's y la amplia red de universidades socias con las que cuenta la Escuela de Arquitectura de la ULPGC, mi planteamiento fue generar un feedback de mi propia experiencia "Staff Mobility Erasmus" e incorporar las relaciones internacionales establecidas a las actividades docentes dentro del aula.

\section{A. Evaluación}

Para que la evaluación fuese ágil y efectiva la realizamos por "observación directa" y por "rúbrica":

La observación directa consistió en prestar atención al comportamiento de los estudiantes durante la realización de cada actividad conjunta: analizando cada acción/reacción de los estudiantes, y con recogida de datos estructurada en base a los cuatro objetivos $\mathrm{OB} 1, \mathrm{OB} 2, \mathrm{OB} 3$ y OB4, detallados en la guía docente.

Esta evaluación nos ha permitido valorar diferentes aspectos como: la actitud y la participación en el aula, así como la aplicación de los conocimientos adquiridos, lo que demuestra si el estudiante ha adquirido o no el nivel de cultura disciplinar necesario, tanto teórico como proyectual, para superar la materia.

Para la evaluación por rúbrica generamos una tabla, donde las filas fueron los seis criterios de evaluación recogidos en la guía docente como resultados de aprendizaje que tendrá que alcanzar el estudiante al finalizar cada tarea:

R1.- El estudiante deberá ser capaz de practicar una lectura del lugar y desplegar un proyecto urbano de pequeña escala, siendo congruente con el lugar mismo, y que suponga una operación que añada complejidad. (Este criterio se cruza con las competencias: CG1; CG6; CG7; CG8; CY2 y CY12)

R2.- Habilidad para trabajar en grupos en el desarrollo de una intervención urbana de pequeña escala con tejido residencial, como respuesta crítica y contenidos disciplinaras que la hagan sólida y coherente con el hecho urbano en el que se insertan. (Se cruza con las competencias: CG7 y CG8)

R3.- Habilidad para el análisis urbano y la síntesis. (Se cruza con las competencias: CG1 y CG8)

R4.- Habilidad para afrontar problemáticas urbanas acotadas a la pequeña escala. (Se cruza con las competencias: CG6; CG7; CG8 y CY2) 
R5.- Habilidad para razonamiento en términos arquitectónicos y urbanos y mostrar una actitud crítica con la realidad. (Se cruza con las competencias: CG8 y CY2)

R6.- Habilidad para decidir los contenidos y las formas de un proyecto urbano de pequeña escala, esto es; las figuras que forman los espacios libres públicos y las arquitecturas, los trazados y los espacios urbanos. (Se cruza con las competencias: CG8; CY2 y CY12)

$\mathrm{Y}$ en cada columna de la rúbrica se cuantificó numéricamente (del 0 al 10) los niveles de calidad alcanzados para cada criterio.

El debate generado en el cierre de las actividades conjuntas fue especialmente útil para evaluar la interacción, y nos ha permitido atestiguar que el nivel de comunicación entre los estudiantes de ambas universidades ha sido bueno. También ha permitido que el estudiante tome conciencia de su propio proceso de pensamiento y lo contaste con otros procedimientos, así como que detecte e intente superar las dificultades encontradas en el proceso de aprendizaje.

\section{B. Resultados}

Los resultados obtenidos al introducir en el proyecto docente de la asignatura "Urbanística, Ordenación de Territorio y Proyectos de Urbanismo II", experiencias y actividades conjuntas con otras universidades del espacio Atlántico, son:

Poner en valor nuestra actividad docente, traspasando las fronteras de nuestro espacio Atlántico, con el uso del Aprendizaje Basado en Proyectos (ABP) y estrategias de evaluación formativa más eficientes ante los nuevos retos.

Ofrecer una transferencia de conocimientos entre universidades y responder a las demandas de formación de nuestro alumnado en el ámbito internacional, al darle la oportunidad al estudiante de que tome contacto con realidades docentes de otras universidades extranjeras, aunque no desarrolle programas de movilidad.

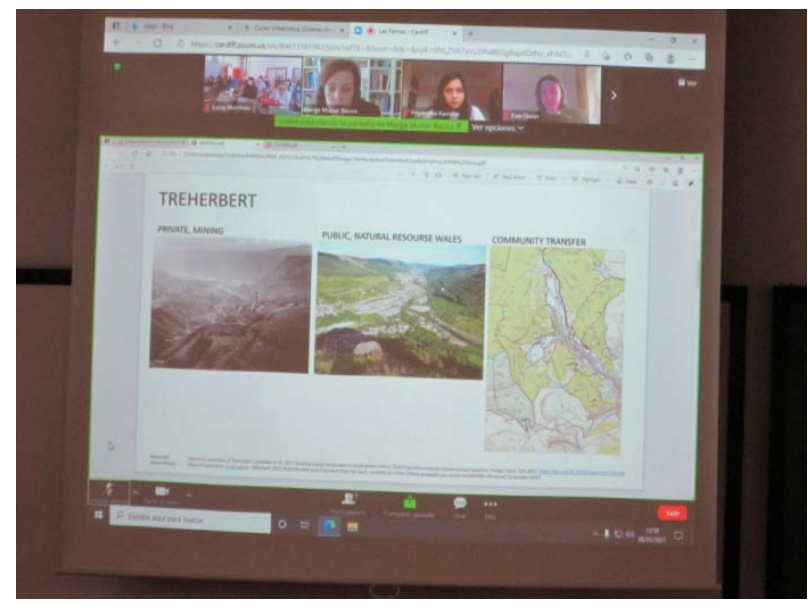

Figura 7: Momento en el que un grupo de estudiantes de la Welsh School of Architecture de Cardiff University presenta su trabajo ante el colectivo de estudiantes de la ULPGC

Apostar por que el estudiante conozca otros modos de hacer, haciéndolos sensibles y receptivos a otras culturas, a otros procedimientos, $\mathrm{y}$ haciéndolos tolerantes con las diferencias.
Evidenciar la importancia de la competencia lingüística de todos los participantes, dentro de un mercado global cada vez más emergente y que repercute en nuestra sociedad.

Garantizar al estudiante una transferencia de conocimientos intercultural, que le propiciará una apertura de miras y le permitirá abordar con mayor madurez y complejidad su futuro profesional, fomentando el razonamiento crítico.

Potenciar el uso de las TIC's en la formación de nuestros estudiantes, buscando el internacionalizar la actividad docente como productor de conocimiento útil, complejo y diverso.

Incentivar a la Escuela de Arquitectura de la ULPGC, a través de su Subdirección de Internacionalización y Cooperación, a desarrollar nuevas alianzas estratégicas con otras universidades, en la búsqueda de fomentar y potenciar la implantación de buenas prácticas en el ámbito de la calidad y la excelencia docente.

Implementar los aspectos que singularizan a la ULPGC en el ámbito universitario, afianzando su Plan de Internacionalización y planificando una agenda de innovación que pueda responder a la misma velocidad que los nuevos cambios.

\section{CONCLUSIONES}

Si bien la ULPGC apuesta por potenciar la internacionalización, de forma paralela se hace necesario poner en valor y reflexionar sobre los modelos pedagógicos, los programas docentes institucionales, y la estructura docente de los Estudios de Arquitectura y Planeamiento en el contexto de la globalización.

El incorporar en los programas docentes metodologías educativas que fortalezcan el vínculo entre universidades socias, permite establecer alianzas estratégicas y potenciar el beneficio mutuo con aquellos PDI de otras universidades que comparten objetivos docentes en el espacio atlántico. Y con ello el estudiante demostrará haber adquirido un nivel de cultura disciplinar, demostrando la comprensión del lugar, el manejo de los conceptos teóricos adquiridos, y la transmisión de conocimientos, al exponerlo y defenderlo adecuadamente no sólo a su tutor y compañeros, sino también a los demás estudiantes y profesores extranjeros involucrados en la actividad.

El objetivo es crear sinergias positivas, optimizando recursos y adquiriendo compromisos que aspiren a una formación más plural y multicultural de nuestro alumnado, y a la creación de escenarios para el desarrollo con un valor compartido, el de la Atlanticidad.

La identificación de los símbolos de la memoria colectiva de estas comunidades y su aplicación en las metodologías educativas de la materia de Urbanística, fortalece un sentimiento identitario y común de nuestro ámbito atlántico, donde la puesta en valor del paisaje y del territorio puede servirnos de revulsivo para mejorar las relaciones interuniversitarias de esta área geográfica. Estas acciones aplicadas a la actividad docente apuestan por una educación de calidad, con equidad e internacionalizada, para ubicarse sólidamente en el escenario de la globalización. Lo que permitirá que nuestros estudiantes egresados se enfrenten a la competencia lingüística del inglés, tan necesaria en estos momentos de globalización y cambio, y tengan una mejor 
capacidad de adaptabilidad en la oferta laboral en un mundo cada vez más cambiante.

Con la aplicación de esta metodología de Aprendizaje Basado en Proyectos Urbanos y dándole una dimensión internacional e institucional, pretendemos cargar de significados la formación de nuestro alumnado. Como nos dice Jean-Louis Déotte "una vida son encuentros, esos encuentros tiene un lugar, la mayor parte del tiempo institucional" (Déotte, 2012, p.136).

En un entorno común, el océano Atlántico, puente de unión entre Europa - África Occidental y América, y una vez comprobado los beneficios de estas metodologías docentes participativas con Aprendizaje Basado en Proyectos Urbanos, nos queda establecer qué otras universidades de este espacio atlántico aún no socias, pueden querer incorporarse e implicarse en esta iniciativa que, de forma conjunta, se involucra en procedimientos metodológicos participativos interculturales, lo que permitirá consolidar a la ULPGC como referente de la internacionalización en el espacio Atlántico.

\section{AgRADECIMIENTOS}

Agradezco la colaboración a la Welsh School of Architecture de la Universidad de Cardiff y en particular a la docente Ph.D. Marga Munar Bouza, por su disponibilidad y participación en el desarrollo de las actividades conjuntas en este curso académico 2020/21. Lo que ha permitido obtener gratificantes resultados en el proceso de aprendizaje de nuestros estudiantes.

\section{REFERENCIAS}

Déotte, J.-L. (2012). La ciudad porosa: Walter Benjamin y la arquitectura. Santiago, Chile: L'Harmattan.

Friedmann, J. (2010). Crossing Borders: Do Planning ideas travel? En P. Healey y R. Upton (Eds.), Crossing Borders: Intenational Exchange and planning practices. (pp. 313-328). Oxford, UK: Routledge.

Luçart, A. (1953). Formes, composition et lois d'armonie. Paris, Francia: Vincent Fréal \& Cie.

Pujolas, P. (2008). 9 ideas clave: el aprendizaje cooperativo. Barcelona, España: Graó.

Valero-García, M. (2007). Las dificultades que tienes cuando haces PBL. En J. A. Marín (Ed.), La Educación Superior hacia la Convergencia Europea: Modelos basados en el aprendizaje. Guipúzcoa, España: Universidad de Mondragón.

Zabala, A. y Arnau, L. (2014). Métodos para la enseñanza de las competencias. Barcelona, España: Graó. 